\section{Scale up isolation of aaptamine for in vivo evaluation indicates its neurobiological activity is linked to the delta opioid receptor}

DOMINICAN

UNIVERSITY

of CALIFORNIA 1890

\section{Introduction}

Opioid receptors belong to the large superfamily of seven transmembrane-spanning (7TM) G protein-coupled receptors (GPCRs). As a class, GPCRs are of fundamental physiologica importance mediating the actions of the majority of known neurotransmitters and hormones. The Mu $(\mu)$, Delta $(\delta)$ and Kappa (MOR, DOR, KOR) opioid receptors are particularly intriguing members of this receptor family as they are the targets involved in many neurobiological diseases such as addiction, pain, stress, anxiety, and depression. To date few marine natural products have been investigated for their neurobiological activities.' One now 1 was the first marne ziconotide (1) from the cone snail Conus used for the treatment of pain marketed under the trade nape Prialt 1 (2004) ${ }^{3}$ and recently Hamman reported that aaptamine (2) is the first marine natural product to show in vivo anti-depressant activity, however no mechanism of action was proposed 1.4 During a separate collaborative screening project we profiled 96 sponge-derived extracts and discovered that demethyl-aaptamine (3) and demethyl (oxy)-aaptamine (4) were selective DOR agonists using an LC-MS based library of an active methenolic extract coll no 92553 FM as shown in Fig. 1. We speculated that the in vivo activity for $\mathbf{2}$ could thus be linked to the DOR target and to test this hypothesis we conducted the following experiments below.
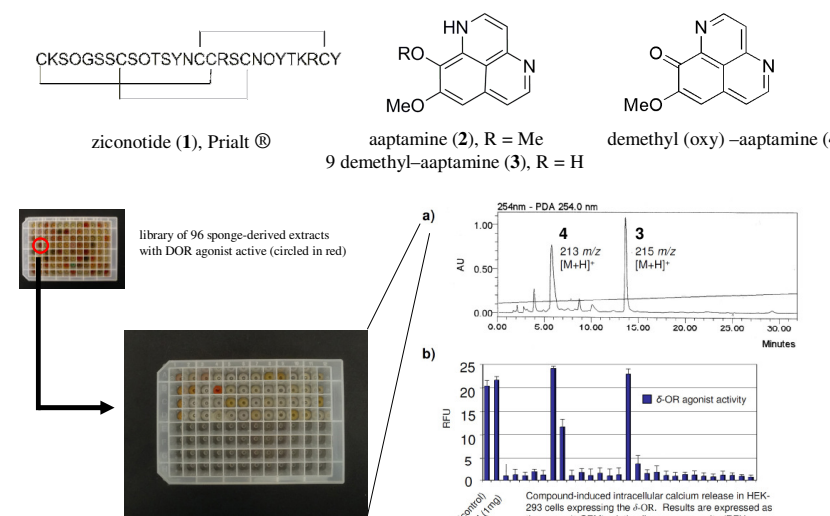

Figure 1. a) LC-MS library with annotations including $\mathrm{m} / z$ ions and b) Comparative DOR agonist activity of the methanol extract LC fractions of coll. no. $92553 \mathrm{FM}$.

\section{Experimental and Results}

Our first step involved obtaining a source of aaptamine (2) for in vitro and in vivo evaluation. Compounds 3-4 were obtained from the sponge Aaptos aaptos (coll. no. 92553) but were devoid of 2. LC-MS analysis of sponge coll. no. 11308 (A. aaptos) indicated $\mathrm{m} / \mathrm{z}$ using a partition scheme shown in Fig. 2. The WB extract was enriched with 2 based on LC-MS data in Fig. 3a and used to scale up it's isolation by HPLC shown in Fig. 3b. Chemical validation of pure 2 was confirmed by LC-MS and ${ }^{1} \mathrm{H}$ NMR data in Fig. 4. This allowed us to screen $\mathbf{2}$ alongside 3-4 and confirm it 's DOR activity in vitro $\left(\mathbf{2}, \mathrm{EC}_{50}=5.1\right.$ $\left.3, \mathrm{EC}_{50}=4.1 ; 4, \mathrm{EC}_{50}=2.3\right)$. In vivo evaluation indicated 2 was an antidepressant in wild type mice in the forced swim test (Fig. 5a, black bars) while having no effect on general locomotion (Fig. 5b). We further found that the antidepressant activity was abolished in genetically modified mice where the DOR gene was knocked out (Fig. Sa, red bars, DOR KO). We also found $\mathbf{2}$ was an anxiolytic in the marble burying test (Fig. 5c). These results indicate the anti-depressant activity previously reported for $\mathbf{2}$ is modulated by it's agonis

Department of Natural Sciences \& Mathematics, Dominican University of California Department of Chemistry \& Biochemistry, University of California, Santa Cruz

${ }^{3}$ Department of Neurology, University of California, San Francisco

†These authors contributed equally to this work
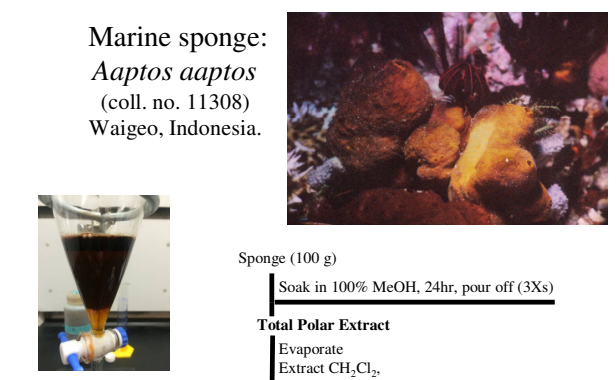

Soak in $100 \%$ MeO
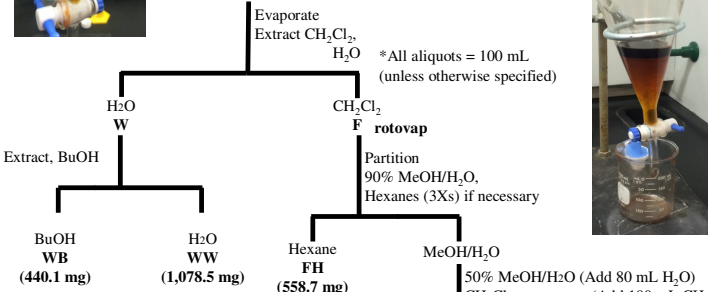

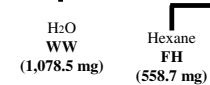

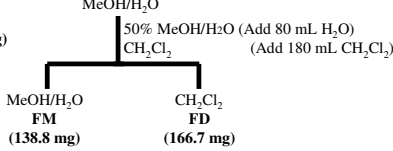

Figure 2. Extraction Scheme Flowchart of coll. no. 11308

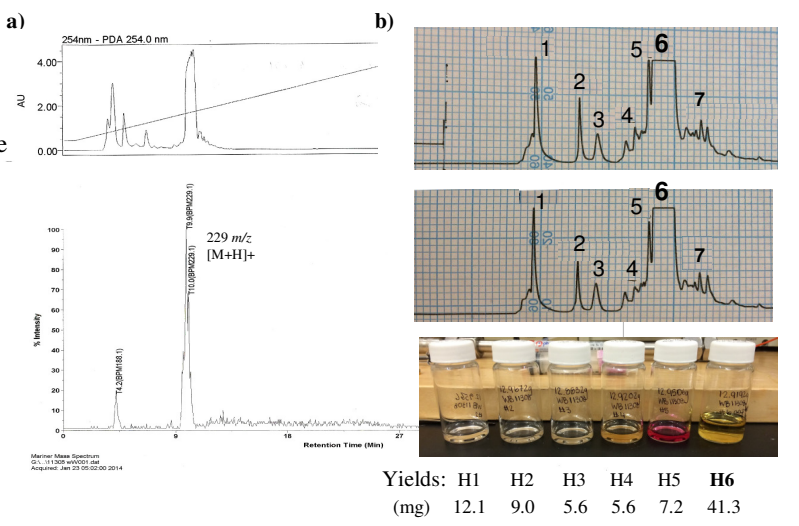

Figure 3. a) Analytical traces LC (top) MS (bottom) of coll. no. 11308 WB an b) Preparative scale up HPLC traces of coll. no. $11308 \mathrm{WB}$ fractions. Gradient: $10 \% \rightarrow 100 \% \mathrm{CH}_{3} \mathrm{CN}(45 \mathrm{~min}) ;[4.0 \mathrm{mg} / 100 \mu \mathrm{l}] \times 30$ injections; $\lambda_{\max }=254 \mathrm{~nm}$; sensitivity $=2.0 \mathrm{AU}$; flow $2.0 \mathrm{ml} / \mathrm{min}$.
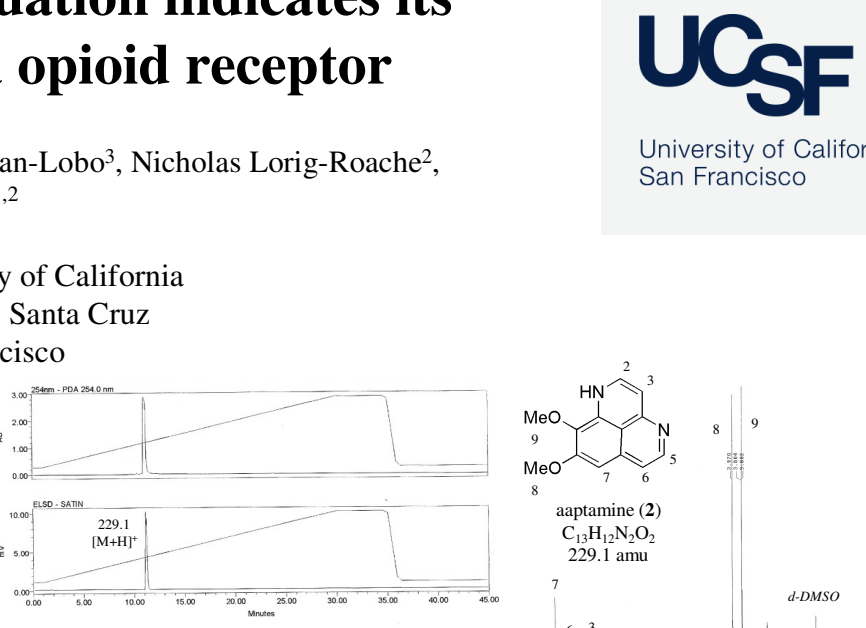

University of California San Francisco
Figure 4. Chemical validation of aptamine (2) using: a) LC-MS-ELSD analysis with annotations including $\mathrm{m} / \mathrm{z}$ ions and b) ${ }^{1} \mathrm{H}$ NMR data of $11308 \mathrm{WB}$ H6.
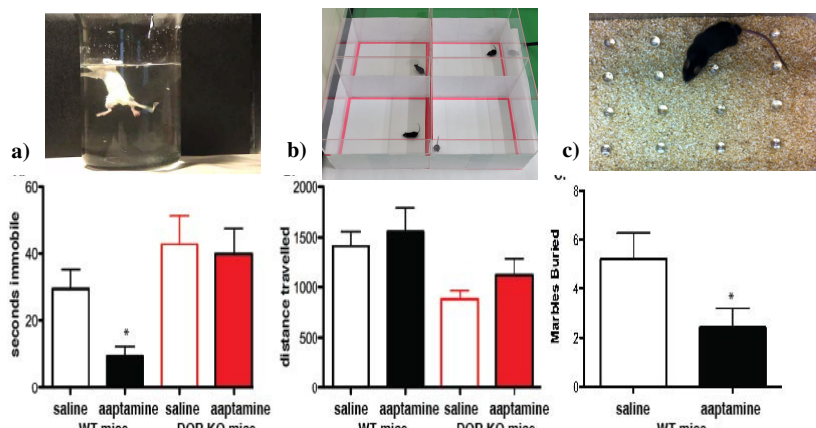

Figure 5. Mice were injected with saline or aaptamine (2, 40mg/kg, i.p.) and subjected to a forced swim test (a), a locomotor test (b) or a marble burying test (c).

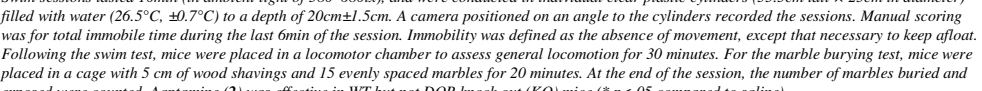

\section{Conclusions}

1) Scale up isolation of aaptamine (2) is best achieved through purification of water soluble extracts. 2) The mechanism of action for the in vivo anti-depressant-like and anxiolytic-like activity of $\mathbf{2}$ is mediated by it's activity on the delta opioid receptor (DOR).

3) These data suggest that $\mathbf{2}$ can represent a novel chemical scaffold for the development of new DOR ligands in neurobiological research.

\section{Acknowledgements}

Financial support was provided by NIH grants RO1 CA 47135 (PC), R01 AA 020401, R01 DA019958, R01 DA0015232, R21 DA031574 (JW), International Cooperative Biodiversity Group (ICBG) grant no. 1 U01TW008160 DA0015232, R21 DA031574 (JW), International Cooperative Biodiversity Group (IC
(JW, TJ), and by the Fletcher Jones Fund of Dominican University of California (TJ).

\section{References}

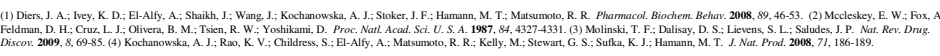

